\title{
Linear Stability in the Inner Heliosphere: Helios Re-evaluated
}

\author{
Kristopher G. Klein ${ }^{1}$ (i), Mihailo Martinović ${ }^{1,2}$ (i), David Stansby ${ }^{3}$ (1) , and Timothy S. Horbury ${ }^{4}$ (i) \\ ${ }^{1}$ University of Arizona, Tucson, AZ, USA \\ ${ }^{2}$ LESIA, Observatoire de Paris, Meudon, France \\ ${ }^{3}$ Mullard Space Science Laboratory, University College London, Holmbury St. Mary, Surrey RH5 6NT, UK \\ ${ }^{4}$ The Blackett Laboratory, Imperial College London, London SW7 2AZ, UK \\ Received 2019 October 30; revised 2019 November 12; accepted 2019 November 13; published 2019 December 23
}

\begin{abstract}
Wave-particle instabilities driven by departures from local thermodynamic equilibrium have been conjectured to play a role in governing solar wind dynamics. We calculate the statistical variation of linear stability over a large subset of Helios I and II observations of the fast solar wind using a numerical evaluation of the Nyquist stability criterion, accounting for multiple sources of free energy associated with protons and helium including temperature anisotropies and relative drifts. We find that $88 \%$ of the surveyed intervals are linearly unstable. The median growth rate of the unstable modes is within an order of magnitude of the turbulent transfer rate, fast enough to potentially impact the turbulent scale-to-scale energy transfer. This rate does not significantly change with radial distance, though the nature of the unstable modes, and which ion components are responsible for driving the instabilities, does vary. The effect of ion-ion collisions on stability is found to be significant; collisionally young wind is much more unstable than collisionally old wind, with very different kinds of instabilities present in the two kinds of wind.
\end{abstract}

Unified Astronomy Thesaurus concepts: Space plasmas (1544); Solar wind (1534); Plasma physics (2089); Plasma astrophysics (1261); Heliosphere (711); Interplanetary turbulence (830); Fast solar wind (1872)

\section{Introduction}

Departures of particle velocity distributions from local thermodynamic equilibrium in the weakly collisional solar wind are frequently observed (see Marsch 2012 and Verscharen et al. 2019 for a review of such measurements) and are associated with wave-particle instabilities, a class of interactions that act to move particle distributions toward equilibrium while simultaneously emitting a variety of plasma waves. A review of the linear and quasilinear theory associated with such instabilities can be found in Gary (1993) and Yoon (2017). The marginal stability thresholds - surfaces in parameter space beyond which an instability has a significant growth ratepredicted by such theories have been shown to constrain observed distributions of parameters in the solar wind and magnetosphere (Kasper et al. 2002; Hellinger et al. 2006; Matteini et al. 2007; Bale et al. 2009; Maruca et al. 2012, 2018; Verscharen et al. 2013; Hellinger \& Trávníček 2014; Chen et al. 2016).

Though these analytic limits are useful, they typically only focus on the effect of a single source of free energy, e.g., proton temperature anisotropy, and exclude other sources, e.g., relative drifts between component distributions or anisotropies of other species. While the presence of many sources of free energy complicates the application of analytic limits, the stability of a linearized system can be determined through the application of the Nyquist instability criterion (Nyquist 1932). In this work, we evaluate the stability of 45,147 solar wind observations made by the Helios spacecraft, a subset of observations with a bi-Maxwellian fit for the helium $(\alpha)$ component described in Stansby et al. (2019), using a numerical implementation of the Nyquist criterion applied to the hot plasma dispersion relation for an arbitrary number of relatively drifting bi-Maxwellian components (Klein et al. 2017b). The Nyquist criterion has been previously applied to hundreds of solar wind measurements made by the Wind spacecraft at 1 au (Klein et al. 2018).
The larger number of observations combined with Helios' coverage of inner heliospheric radial distances provides an excellent opportunity to characterize how instabilities behave under different plasma and solar wind conditions.

After reviewing the numerical methodology and the data set, we survey the calculated linear stability as a function of solar wind and plasma parameters. More than $87 \%$ of intervals were found to be linearly unstable, slightly more than expectations from simple stability thresholds. The median growth rates of the unstable intervals are considerable, usually around $20 \%-30 \%$ of the turbulent cascade rate. There is no significant change in the fraction of unstable modes with increasing distance in the selected data set. The character of the unstable modes does change as a parcel of plasma moves further from the Sun, with the $\alpha$ component playing a more significant role in driving unstable behavior at larger distances. Highly collisional intervals are much less unstable, with the $\alpha$ component acting as the sole source of free energy for the most collisionally processed intervals.

\section{Data and Methodology}

\subsection{Methodology}

Instead of using canonical parametric stability thresholds (Gary et al. 1998; Kasper et al. 2002; Hellinger et al. 2006; Bale et al. 2009), which typically depend on only a single source of free energy, we determine the linear stability for a set of measured parameters by applying a numerical implementation of the Nyquist instability criterion (plumage, described in Klein et al. 2017b). We numerically evaluate a contour integral over the inverse of the hot plasma dispersion relation $\operatorname{det}[\mathcal{D}(\omega, \boldsymbol{k} ; \mathcal{P})]$,

$$
W_{n}(\boldsymbol{k}, \mathcal{P})=\frac{1}{2 \pi i} \oint \frac{d \omega}{\operatorname{det}[\mathcal{D}(\omega, \boldsymbol{k} ; \mathcal{P})]}
$$


The real and imaginary components of the complex frequency $\omega$ are $\omega_{\mathrm{r}}$ and $\gamma, \boldsymbol{k}$ is the wavevector, and $\mathcal{P}$ is the set of dimensionless plasma parameters describing the system, including, e.g., plasma $\beta$ and $T_{\perp} / T_{\|}$. As shown by Nyquist (1932), the integer solution of this integral $W_{n}(\boldsymbol{k}, \mathcal{P})$ represents the number of linearly unstable modes supported by the dispersion relation for a particular wavevector $\boldsymbol{k}$ at a particular point in parameter space $\mathcal{P}$. As demonstrated in Klein et al. (2017b), by iteratively increasing the lower bound of the integration path from $\gamma=0$ to a finite $\gamma$, the maximum growth rate $\gamma^{\max }(\boldsymbol{k}, \mathcal{P})$ can be determined as the value of $\gamma$ for which $W_{n}(\boldsymbol{k}, \mathcal{P})$ is non-zero, but $W_{n}(\boldsymbol{k}, \mathcal{P})=0$ for any larger value of $\gamma$.

This calculation can be performed for any dispersion relation that can be numerically evaluated; for this work, we focus on the case of a collection of an arbitrarily number of bi-Maxwellian components drifting with respect to one another, and use the dispersion relation numerically evaluated by PLUME (Klein \& Howes 2015). Each component $j$ has a unique temperature parallel $\left(T_{\|, j}\right)$ and perpendicular $\left(T_{\perp, j}\right)$ to the local magnetic field $\boldsymbol{B}$, a density $n_{j}$, and a bulk velocity $\boldsymbol{U}_{j}$, as well as the intrinsic mass $m_{j}$ and charge $q_{j}$ associated with the component. For this calculation, we use a reference component to normalize these quantities and produce six dimensionless parameters to describe each component: $T_{\perp, j} / T_{\|, j}, T_{\|, \text {ref }} / T_{\|, j}, \Delta v_{j, \text { ref }} / v_{A, \text { ref }}, n_{j} / n_{\text {ref }}, m_{j} / m_{\text {ref }}$, and $q_{j} / q_{\text {ref }}$. The Alfvén velocity is calculated using only the reference mass density $v_{A \text {,ref }}=B / \sqrt{4 \pi n_{\text {ref }} m_{\text {ref }}}$, and $\Delta v_{j \text {,ref }}$ is the magnetic-field-aligned difference in drift speeds between the reference species and component $j$. The global dimensionless parameters $\beta_{\|, \text {ref }}=8 \pi n_{\text {ref }} T_{\|, \text {ref }} / B^{2}$ and the reference thermal velocity normalized by the speed of light $w_{\|, \text {ref }} / c$ complete our description of the system. Timescales are normalized to the reference cyclotron frequency $\Omega_{\text {ref }}=$ $q_{\mathrm{ref}} B / m_{\mathrm{ref}} c$.

\subsection{Data}

While this numerical method has previously been applied to hundreds of intervals from the Wind spacecraft at 1 au (Klein et al. 2018), a statistical assessment of linear stability as a function of solar wind and plasma parameters requires analysis of a larger set of data. Additionally, using measurements closer to the Sun than 1 au assists the study of radial variations in inferred stability. For these reasons, we use the recent reanalysis of Helios proton core (Stansby et al. 2018) and helium (Stansby et al. 2019) measurements, which produced parallel and perpendicular temperatures, densities, and drift speeds for the proton core and helium components. This processing of the Helios data did not include a secondary proton, or beam, component. Due to the method used to extract helium temperature, this data set has a bias toward faster solar wind, and does not have a uniform coverage of all radial distances; this bias is shown in the top row of Figure 3. All intervals are of the same length, that of the integration time for the Helios onboard electrostatic analyzer, $40.5 \mathrm{~s}$.

The dispersion relation $\operatorname{det}\left[\mathcal{D}\left(\omega / \Omega_{p}, \boldsymbol{k} \rho_{p} ; \mathcal{P}\right)\right]$ for each of the 45,147 measured intervals in this data set can be described using

$$
\begin{aligned}
\mathcal{P}= & \left\{\beta_{\|, p}, \frac{w_{\|, p}}{c}, \frac{T_{\perp, p}}{T_{\|, p}}, \frac{T_{\perp, \alpha}}{T_{\|, \alpha}}, \frac{T_{\perp, e}}{T_{\|, e}}, \frac{T_{\|, p}}{T_{\|, \alpha}}, \frac{T_{\|, p}}{T_{\|, e}},\right. \\
& \left.\frac{n_{\alpha}}{n_{p}}, \frac{n_{e}}{n_{p}}, \frac{\Delta v_{\alpha, p}}{v_{A, p}}, \frac{\Delta v_{e, p}}{v_{A, p}}\right\},
\end{aligned}
$$

as well as the intrinsic mass and charge ratios. The subscripts $p, \alpha$, and $e$ indicate proton, helium, and electron quantities. Given the observed complexity of electron structure and the comparatively simple, single bi-Maxwellian model used in this study, we refrain from any analysis of electron-scale instabilities, and set $n_{e} / n_{p}$ and $\Delta v_{e, p} / v_{A, p}$ to enforce zero net current and quasineutrality. We also assume an isotropic electron distribution $T_{\perp, e} / T_{\|, e}=1$ and set $T_{p}=\left(2 T_{\perp, p}+T_{\|, p}\right) / 3=T_{e}$. This leaves us with a sevendimensional set of varying parameters for our stability model:

$$
\mathcal{P}=\left\{\beta_{\|, p}, \frac{w_{\|, p}}{c}, \frac{T_{\perp, p}}{T_{\|, p}}, \frac{T_{\perp, \alpha}}{T_{\|, \alpha}}, \frac{T_{\|, p}}{T_{\|, \alpha}}, \frac{n_{\alpha}}{n_{p}}, \frac{\Delta v_{\alpha, p}}{v_{A, p}}\right\} .
$$

For each interval with a good $\alpha$ fit in Stansby et al. (2019), values for $\mathcal{P}$ are calculated by combining the $\alpha$ properties with bi-Maxwellian proton-core fits from Stansby et al. (2018), supplemented by solar wind parameters from the publicly available Helios Data Archive (NASA 2018).

\subsection{Analysis}

For each interval, we evaluate the growth rate $\gamma^{\max }\left(\boldsymbol{k} \rho_{p}, \mathcal{P}\right) /$ $\Omega_{p}$ for wavevectors normalized to the proton gyroradius, $\rho_{p}=\sqrt{2 T_{\perp, p} / m_{p}}$, covering a logarithmically spaced grid, $k_{\perp} \rho_{p} \in\left[10^{-3}, 3\right]$ and $k_{\|} \rho_{p} \in\left[10^{-2}, 3\right]$, focusing on instabilities that arise at ion scales. Four examples of such wavevector grids are shown in the left column of Figure 1, with the time of measurement and associated dimensionless parameters provided in Table 1.

For each interval, we determine the largest growth rate within the specified wavevector range, denoted $\gamma^{\max }(\mathcal{P}) / \Omega_{p}$, and the associated wavevector $\boldsymbol{k}^{\max } \rho_{p}$. While there are frequently several different types of instabilities associated with a particular interval- see rows two and four of Figure 1the fastest growing mode will dominate the system's dynamics. Using $\gamma^{\max }(\mathcal{P}) / \Omega_{p}$ and $\boldsymbol{k}^{\max } \rho_{p}$, we calculate the real frequency of the fastest growing mode $\omega_{\mathrm{r}}\left(\boldsymbol{k}^{\max } \rho_{p}, \mathcal{P}\right) / \Omega_{p}$ by searching for zeros in $\operatorname{det}\left[\mathcal{D}\left(\omega / \Omega_{p}, \boldsymbol{k} \rho_{p}, \mathcal{P}\right)\right]$ near $\gamma^{\max }(\mathcal{P}) / \Omega_{p}$. With $\omega_{r}^{\max } / \Omega_{p}$ determined, we calculate the linear eigenfunctions for electromagnetic field and plasma fluctuations associated with this normal mode, derived quantities including magnetic field polarization (Gary 1993; Krauss-Varban et al. 1994; Schwartz et al. 1996; Klein 2013), and the power absorption or emission per wave-period due to the $j$ th plasma component (Stix 1992; Quataert 1998; Klein et al. 2017a),

$$
P_{j}=\frac{\gamma_{j}}{\omega_{\mathrm{r}}}=\frac{\boldsymbol{E}^{*} \cdot \underline{\underline{\chi}}_{j}^{a} \cdot \boldsymbol{E}}{4 W_{\mathrm{EM}}},
$$

where $\boldsymbol{E}$ and $\boldsymbol{E}^{*}$ are the perturbed electric field and its complex conjugate, $\underline{\underline{\chi}}_{s}^{a}$ is the anti-Hermitian part of the linear susceptibility tensor for component $j$ evaluated at the normalmode frequency $\omega_{\mathrm{r}}$, and $W_{\mathrm{EM}}$ is the electromagnetic wave 


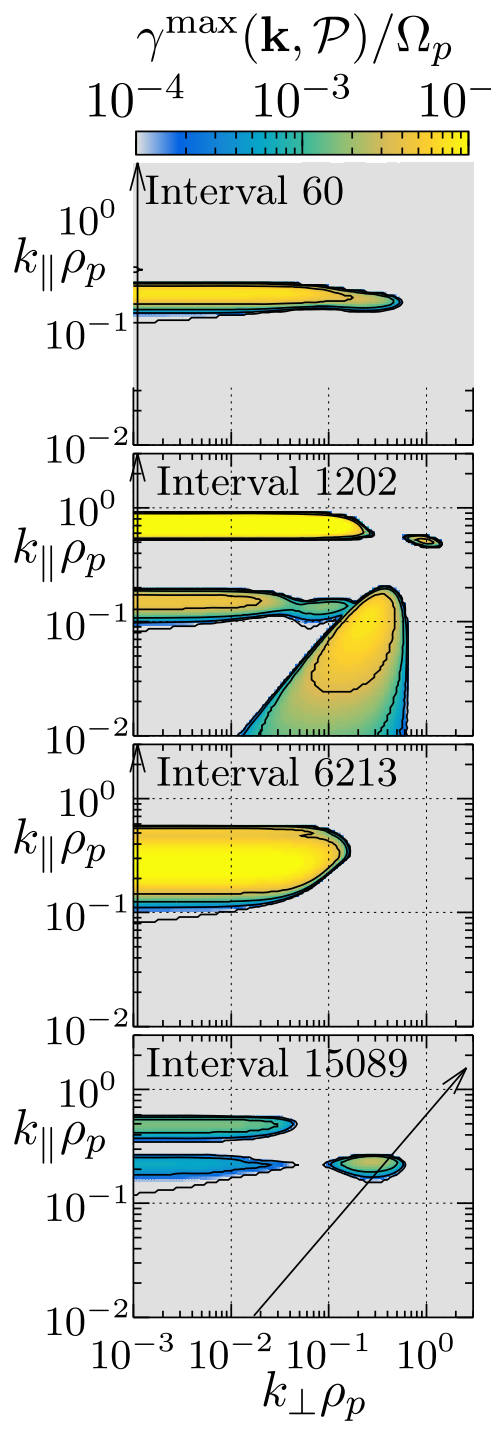

$$
-2
$$$$
\begin{aligned}
& -P^{\text {total }}<0 \\
& -P^{\text {total }}>0
\end{aligned}
$$
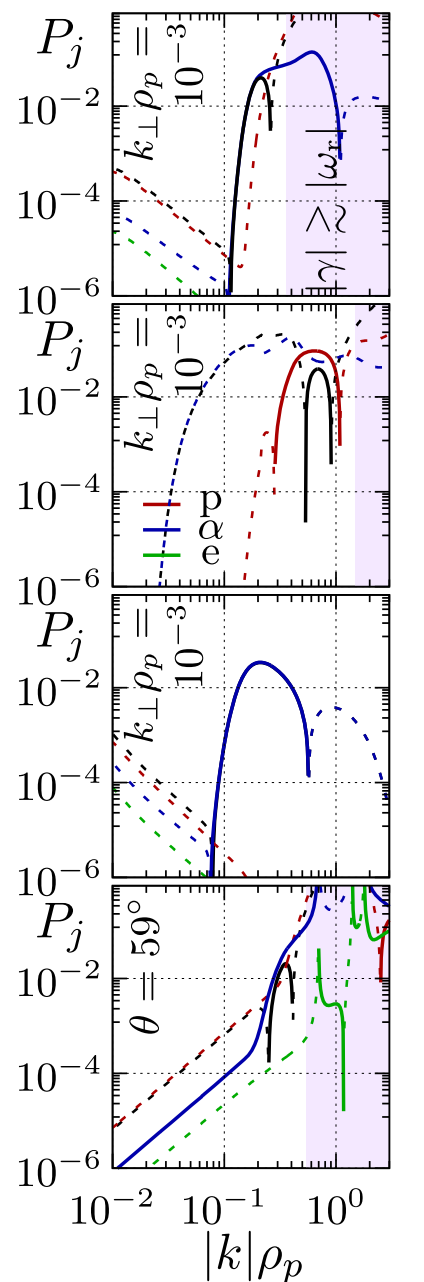

Figure 1. The maximum growth rate $\gamma^{\max }(\boldsymbol{k}, \mathcal{P}) / \Omega_{p}$ (left column) and the power absorption (dashed lines) or emission (solid) for the dispersion surface of the fastest growing mode evaluated along the arrows in the left column (right). The contributions to the total power transfer (black) from the protons (red), $\alpha$ s (blue), and electrons (green) are separated to identify which component drives unstable behavior. Shaded regions indicate wavevectors for which the assumption $\gamma \ll \omega_{\mathrm{r}}$ is violated for the selected dispersion surface.

energy. The normal mode's growth rate is $\gamma / \omega_{\mathrm{r}}=\sum_{j} P_{j}$; $P_{j}<0 \quad\left(P_{j}>0\right)$ denotes energy transfer from plasma component $j$ to the waves (waves to plasma component $j$ ). The wavevector region where the underlying assumption for calculation of the linear dispersion relation (i.e., $\gamma<\omega_{\mathrm{r}}$ ) breaks down is shaded in purple in the right column of Figure 1.

The first row of Figure 1 illustrates a typical parallel resonant instability, with $k_{\perp} \ll k_{\|}$and $k_{\|} \rho_{i} \lesssim 1$. For this case, the wave resonantly interacts with the $\alpha$ component; as seen in the sign of $P_{\alpha}$ in right column, the power emitted by the $\alpha \mathrm{s}$ is, for a narrow range of wavevectors, greater than the power absorbed by the protons, producing a net transfer of energy from the charged particles to the electromagnetic field. ${ }^{5}$ The fastest

\footnotetext{
5 In all intervals considered, the electrons contributed negligibly to the overall energy transfer.
}

growing mode propagates along $\boldsymbol{B}$, and the polarization of the magnetic field fluctuation in the wave frame at the peak growth rate is left-handed, indicating that the unstable mode is an ioncyclotron wave rather than a fast-mode-like wave.

In the second row of Figure 1, we illustrate an example where protons emit rather than absorb power. Here, the power absorption by the $\alpha$ component is significant for the wavevectors where $P_{p}<0$, limiting the total growth rate of the unstable mode compared to a proton and electron description. This mode propagates antiparallel to $\boldsymbol{B}$, but in the wave frame the magnetic field polarization is left-handed, again representing an ion-cyclotron type of wave.

The interval shown in the third row of Figure 1 represents a right-handed, parallel-propagating instability, driven by the relative drift between ion components. Neither the protons nor electrons gain energy from or lose energy to the wave, while the $\alpha$ component emits power over a relatively broad region of wavevectors compared to the left-handed waves in rows 1 and 2. In a minority of unstable cases, illustrated in the bottom row, an oblique mode with $k_{\perp} \gtrsim k_{\|}$is the fastest growing mode. Such modes, which represent some version of either the oblique firehose or mirror instability, have magnetic field polarizations near zero and lower values for $\omega_{\mathrm{r}}$ than the parallel instabilities.

\section{Statistical Analysis of Helios Intervals}

From 45,147 intervals, we find that 39,695 (87.9\%) are linearly unstable with $\gamma^{\max }(\mathcal{P}) / \Omega_{p} \geqslant 10^{-4}$. This decreases to $37,760(83.6 \%)$ and $30,963(68.6 \%)$ for thresholds of $\gamma^{\max }(\mathcal{P}) / \Omega_{p} \geqslant 10^{-3}$ and $10^{-2}$. Evaluating the stability of the same intervals using parametric thresholds derived using only proton temperature anisotropy (e.g., Verscharen et al. 2016), and thus neglecting the effects of the $\alpha$ temperature anisotropy and the relative drift between the ion components, we find $82.1 \%, 79.4 \%$, and $69.6 \%$ of the intervals surpass the thresholds $\gamma / \Omega_{p}=10^{-4}, 10^{-3}$, and $10^{-2}$. We hypothesize that this slight decrease in the number of very unstable intervals may be due to additional power absorption by the $\alpha$ s, as shown in row 2 of Figure 1.

We determine which of the two resolved ion components drives the fastest growing mode using $P_{j}$. In most cases, either $P_{p}<0$ and $P_{\alpha} \gtrsim 0$ (30,535 intervals) or $P_{\alpha}<0$ and $P_{p} \gtrsim 0$ (7845 intervals), leaving no ambiguity about which component is emitting power (the component with $P_{j}<0$ ) and which component is absorbing power $\left(P_{j}>0\right)$. There are a small number of cases (1315 intervals) where $P_{p}$ and $P_{\alpha}$ are less than zero and are within an order of magnitude of each other; for these cases, we define the instability as being driven jointly by both ion components.

To further quantify the distribution of growth rates, we plot in Figure 2 the fraction of intervals that have $\gamma^{\max }(\mathcal{P}) / \Omega_{p}$ greater than a specified value, $\gamma_{0} / \Omega_{p}$. From this variant of a cumulative distribution function, we see that most of the unstable modes with $P_{p}<0$ have $\gamma^{\max }(\mathcal{P}) / \Omega_{p} \approx 10^{-2}$, while most of the unstable modes with $P_{\alpha}<0$ are more slowly growing. Overall, $(66,50,33,10) \%$ of the 45,147 intervals satisfy $\gamma^{\max }(\mathcal{P})>(0.011,0.022,0.033,0.055) \Omega_{p}$.

We rescale the growth rates using other characteristic timescales. Renormalizing the growth rates to $\omega_{\mathrm{r}}$, characterizing how quickly the instability can grow compared to the wave's propagation, yields a similar distribution to that normalized to $\Omega_{p}$, with $(66,50,33,10) \%$ of the total intervals 
Table 1

Key Dimensionless Parameters Associated with Four Selected Intervals Illustrated in Figure 1 and the Derived Wave Properties

\begin{tabular}{|c|c|c|c|c|c|c|c|c|c|}
\hline Interval \# & \multicolumn{2}{|c|}{ Date } & $\beta_{\|, p}$ & $w_{\|, p} / c$ & $T_{\perp, p} / T_{\|, p}$ & $T_{\|, p} / T_{\|, \alpha}$ & $T_{\perp, \alpha} / T_{\|, \alpha}$ & $n_{\alpha} / n_{p}$ & $\overline{\Delta v_{\alpha, p} / v_{A, p}}$ \\
\hline 60 & \multicolumn{2}{|c|}{1975 Feb 8 00:41:28 } & 0.642 & $8.45 \times 10^{-5}$ & 0.547 & 0.690 & 3.38 & 0.0315 & -0.0658 \\
\hline 1202 & \multicolumn{2}{|c|}{1975 Feb 9 17:10:25 } & 0.240 & $1.18 \times 10^{-4}$ & 3.10 & 0.0353 & 1.49 & 0.201 & -0.0136 \\
\hline 6213 & \multicolumn{2}{|c|}{1975 Feb 15 19:08:20 } & 0.239 & $1.17 \times 10^{-4}$ & 2.26 & 0.0329 & 0.462 & 0.0784 & 0.673 \\
\hline 15,089 & \multicolumn{2}{|c|}{1976 Mar 19 14:17:10 } & 1.86 & $2.65 \times 10^{-4}$ & 0.606 & 0.252 & 1.00 & 0.0524 & -1.06 \\
\hline Interval \# & $\omega_{r}^{\max } / \Omega_{p}$ & \multicolumn{2}{|c|}{$\gamma^{\max } / \Omega_{p}$} & $k_{\perp}^{\max } \rho_{p}$ & $k_{\|}^{\max } \rho_{p}$ & $P_{p}$ & \multicolumn{2}{|c|}{$P_{\alpha}$} & Polarization \\
\hline 60 & 0.210 & \multicolumn{2}{|c|}{$7.10 \times 10^{-3}$} & $1.00 \times 10^{-3}$ & 0.193 & $1.81 \times 10^{-3}$ & \multicolumn{2}{|c|}{$-3.54 \times 10^{-2}$} & -1.00 \\
\hline 1202 & -0.606 & \multicolumn{2}{|c|}{$2.33 \times 10^{-2}$} & $1.00 \times 10^{-3}$ & 0.684 & $-9.23 \times 10^{-2}$ & \multicolumn{2}{|c|}{$5.60 \times 10^{-2}$} & -1.00 \\
\hline 6213 & 0.421 & \multicolumn{2}{|c|}{$1.22 \times 10^{-2}$} & $1.00 \times 10^{-3}$ & 0.262 & $1.08 \times 10^{-7}$ & \multicolumn{2}{|c|}{$-2.92 \times 10^{-2}$} & 1.00 \\
\hline 15,089 & -0.142 & \multicolumn{2}{|c|}{$2.81 \times 10^{-3}$} & $2.80 \times 10^{-1}$ & 0.238 & $2.25 \times 10^{-2}$ & \multicolumn{2}{|c|}{$-4.26 \times 10^{-2}$} & -0.14 \\
\hline
\end{tabular}

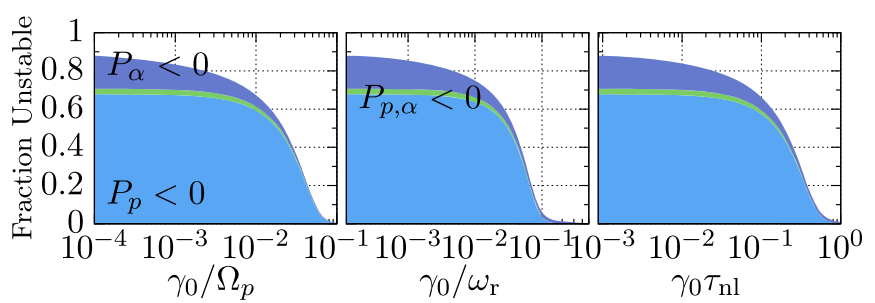

Figure 2. The fraction of intervals that have $\gamma^{\max }(\mathcal{P}) T$ greater $\gamma_{0} T$, for $T=\Omega_{p}^{-1}$ (left), $\omega_{\mathrm{r}}^{-1}$ (center), and $\tau_{\mathrm{nl}}$ (right). Shading indicates whether protons (blue) or $\alpha$ s (purple) are the primary power emitter, or both ion components contribute (green).

satisfying $\gamma^{\max }(\mathcal{P})>(0.021,0.038,0.057,0.088) \omega_{\mathrm{r}}$. We note that $6 \%$ of the total intervals have $\gamma^{\max }(\mathcal{P}) / \omega_{\mathrm{r}}>0.1$; such intervals have reached the edge of validity of linear theory.

Renormalizing $\gamma^{\max }(\mathcal{P})$ to an estimate for the critically balanced nonlinear cascade rate $\tau_{\text {nl }} \sim \tau_{\text {lin }} \sim \omega_{\text {Alfvén }}^{-1}$ (Goldreich $\&$ Sridhar 1995; Mallet et al. 2015) at scale $k=\left|\boldsymbol{k}^{\mathrm{max}}\right|$,

$$
\tau_{\mathrm{nl}}=\frac{\rho_{p}}{v_{A}}\left(k_{0} \rho_{p}\right)^{-1 / 3}\left(\left|\boldsymbol{k}^{\max }\right| \rho_{p}\right)^{-2 / 3}
$$

characterizes how quickly the instability can grow compared to the nonlinear turbulent transfer of energy. To estimate the outer scale $k_{0}$, we subdivide Helios magnetometer measurements into intervals of 12 hours, perform a Fourier transform to construct turbulent power spectra, and identify the spectral break between the energy-containing and inertial ranges. We average the power spectra into bins as a function of radial distance and solar wind speed. Inside each distance-and-speed bin, the spectral break frequency $f_{\text {break }}$ is found by a linear fitting of the averaged spectrum on a logarithmic scale using two free parameters, $f_{\text {break }}$ and the amplitude of the spectrum at $f=10^{-3} \mathrm{~Hz}$, assuming the spectrum has slopes of $f^{-1}$ and $f^{-5 / 3}$ above and below $f_{\text {break }}$. Using these fitted values of $f_{\text {break }}\left(R, v_{\mathrm{sw}}\right)$, an outer scale for each interval is calculated, $k_{0}=2 \pi f_{\text {break }} / v_{\mathrm{sw}}$, which combined with $\left|\boldsymbol{k}^{\mathrm{max}}\right|$ in Equation (5) yields $\tau_{\mathrm{nl}}$. For all intervals, $(66,50,33,10) \%$ have $\gamma^{\max }(\mathcal{P}) \tau_{\mathrm{nl}}>(0.102,0.197,0.297,0.496)$, indicating that for many intervals, the growth rate is within a factor of a few of the turbulent cascade rate, and therefore the instability may grow quickly enough to impact the turbulent scale-to-scale transfer of energy.

As was found at $1 \mathrm{au}$, parallel instabilities are much more common than oblique instabilities. We define in this work a parallel instability as an interval where the fastest growing mode has $k_{\perp}=10^{-3}$, the smallest perpendicular wavevector resolved on our wavevector grid; oblique instabilities are those with $k_{\perp}>10^{-3} ; 39,602$ intervals have parallel fastest growing modes, while 93 intervals have oblique fastest growing modes. Note that this definition of parallel and oblique varies from that presented in Klein et al. (2018), where the authors used the wavevector distribution of instabilities combined with the excess parallel or perpendicular pressure to classify intervals as kinetic, firehose, or mirror unstable. We identify many more than 94 intervals with oblique instabilities, e.g., interval 1202 in Figure 1, but in most cases there exists a faster growing parallel instability. As the fastest growing mode dominates the plasma's evolution, we find the definition employed here more physically meaningful than that used in Klein et al. (2018); of the 309 intervals studied in that work, 166 of which were unstable, only eight have an oblique fastest growing mode.

In Figure 3 we subdivide the data set as a function of radial distance from the Sun $R$, solar wind speed $v_{\mathrm{sw}}$, Coulomb number $N_{\alpha, p}=\nu_{\alpha, p} R / v_{\mathrm{sw}}$ (Feldman et al. 1974; Neugebauer 1976), and excess parallel pressure (Kunz et al. 2015; Chen et al. 2016)

$$
\Lambda_{\mathrm{F}}=\sum_{j} \frac{\beta_{\|, j}-\beta_{\perp, j}}{2}+\frac{\sum_{j} n_{j} m_{j}\left|\Delta u_{j}\right|^{2}}{\left(\sum_{j} n_{j} m_{j}\right) v_{A}^{2}} .
$$

For the calculation of $N_{\alpha, p}$ we use the proton- $\alpha$ collision frequency $\nu_{\alpha, p}$ described in Hernandez et al. (1987). For the calculation of $\Lambda_{\mathrm{F}}, \Delta u_{j}$ is the difference between the bulk velocity of component $j$ and the center-of-mass velocity. In Figure 4, we analyze the data as a function of proton temperature anisotropy $T_{\perp, p} / T_{\|, p}, \alpha$ temperature anisotropy $T_{\perp, \alpha} / T_{\|, \alpha}$, scalar temperature disequilibrium $T_{\alpha} / T_{p}$, and normalized drift speed $\Delta v_{\alpha, p} / v_{A, p}$. The rows of Figures 3 and 4 are organized as follows: top row, the number of intervals associated with the abscissa coördinate; second row, the fraction of intervals found to be linearly unstable segregated by angle of $\boldsymbol{k}^{\max } \rho_{p}$ and $P_{j}$ (purple is parallel and $P_{\alpha}<0$, blue parallel and $P_{p}<0$, green parallel and both $P_{p}<0$ and $P_{\alpha}<0$, orange oblique and $P_{\alpha}<0$, and red oblique and $P_{p}<0$ ); third row, the distribution of wave-frame magnetic field polarizations (red is right-handed, blue left-handed, gray nearly zero polarization); fourth row, the distribution of propagation direction in the plasma frame (pink is anti-sunward, yellow is sunward); last row, the median value of $\gamma^{\max }(\mathcal{P}) / \Omega_{p}$ (blue), 


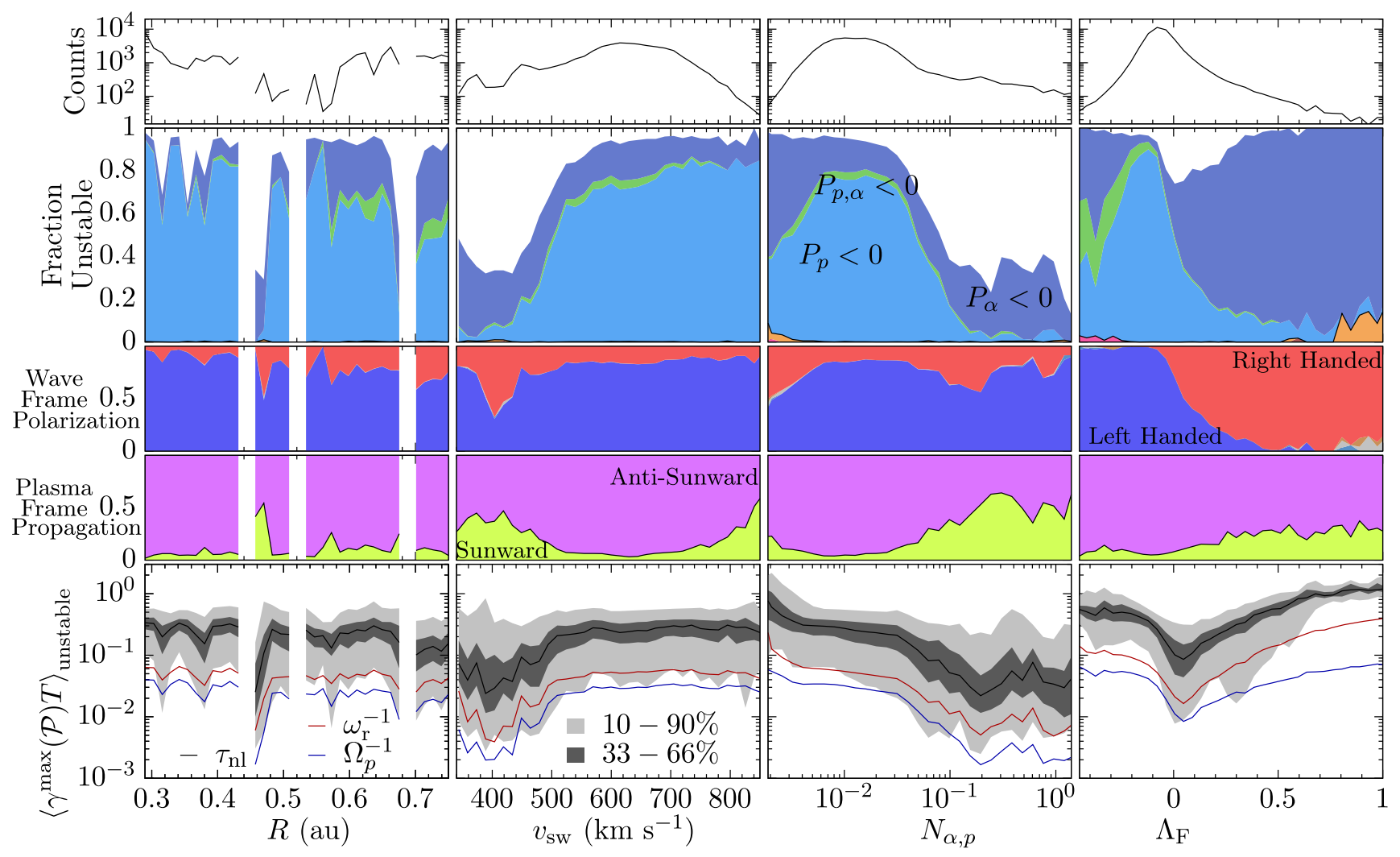

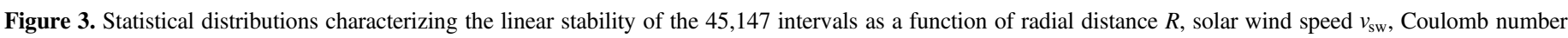
$N_{\alpha, p}$, and excess parallel pressure $\Lambda_{\mathrm{F}}$. The layout is described in the text.

$\gamma^{\max }(\mathcal{P}) / \omega_{\mathrm{r}}$ (red), and $\gamma^{\max }(\mathcal{P}) \tau_{\mathrm{nl}}$ (black) drawn from the subset of unstable intervals; the light and dark gray shading indicates the ranges that enclose $(10,90) \%$ and $(33,66) \%$ of the values of $\gamma^{\max }(\mathcal{P}) \tau_{\mathrm{nl}}$.

The fraction of linearly unstable intervals is relatively constant as a function of radial distance from the Sun. ${ }^{6}$ With increasing distance, the fraction of intervals where the $\alpha$ emission of power drives the fastest growing mode increases from a few per cent near 0.3 au to more than $20 \%$ at 0.7 au. A similar transition with increasing distance is seen from uniformly left-handed waves to a minority of right-handed waves. The interval between 0.46 and 0.48 au is an unusual subset of the data, entirely composed of slow wind, as opposed to all other distances, which are dominated by fast wind intervals. The median growth rate, normalized to $\Omega_{p}^{-1}, \omega_{\mathrm{r}}^{-1}$, or $\tau_{\text {nl }}$, is relatively constant with distance, decreasing by less than a factor of two over those radial distances.

More significant variations arise with solar wind speed; $90 \%$ of fast wind intervals with $v_{\mathrm{sw}}>550 \mathrm{~km} \mathrm{~s}^{-1}$ are unstable with a median growth rate of $\left\langle\gamma^{\max }(\mathcal{P}) \tau_{\mathrm{nl}}\right\rangle \approx 0.3$ and are predominantly left-handed. Slower wind is much less unstable, with slower growth rates $\left\langle\gamma^{\max }(\mathcal{P}) \tau_{\text {nl }}\right\rangle \approx 0.03$. A larger fraction of the unstable modes in the slow wind propagate toward the Sun. There is a noticeable uptick in sunward propagation for wind with $v_{\mathrm{sw}}>700 \mathrm{~km} \mathrm{~s}^{-1}$, though there are relatively few measurements satisfying this criterion. For slower wind, more of the unstable intervals are driven by $\alpha$ s than protons.

Similar variations are seen with Coulomb number. The collisionally oldest wind, intervals with $N_{\alpha, p} \gtrsim 1$, has fewer

\footnotetext{
6 The three white stripes in the left column of Figure 3 represent distances with too few measurements to enable a statistical study.
}

unstable intervals and these instabilities are driven by $\alpha$ s rather than protons and are evenly divided between sunward and antisunward propagation. In collisionally younger wind, a majority of intervals are unstable with protons serving as the main instability driver. In the collisionally youngest wind, intervals with $N_{\alpha, p} \lesssim 5 \times 10^{-3}$, the $\alpha$ s drive approximately half of the unstable modes and the median growth rate is enhanced. This variation, not observed in the fastest solar wind, may be a signature of helium driven far out of thermal equilibrium in the near-Sun region of preferential minor ion heating hypothesized by Kasper et al. (2017) and Kasper \& Klein (2019), a conjecture that will be investigated with observations closer to the Sun.

Variations with $\Lambda_{\mathrm{F}}$ are stark. For intervals with $\Lambda_{\mathrm{F}}>0$, representing intervals with excess parallel pressure, the instabilities are generally right-handed and driven by the $\alpha$ component. This is expected: as $T_{\perp p} / T_{\| p}$ is generally greater than one, the excess parallel pressure is driven by the drifting $\alpha$ component. For $\Lambda_{\mathrm{F}} \approx 1$, nearly $5 \%$ of the intervals have an oblique fastest growing modes. For $\Lambda_{\mathrm{F}}<0$, representing cases with greater perpendicular than parallel pressure, the instabilities are left-handed, with contributions from protons, $\alpha$ s, or both ion components to the unstable growth. For significant deviations from $\Lambda_{\mathrm{F}}=0$ the median growth rates are enhanced.

Unsurprisingly, intervals with large proton temperature anisotropies are associated with instabilities primarily driven by protons; the modes are left-handed with anti-sunward propagation. Fewer intervals with $T_{\perp, p} / T_{\|, p}<2$ are unstable, and of those that are, a larger fraction have both right-handed magnetic fluctuations and (plasma frame) sunward propagation. These intervals also have generally slower growth rates than large anisotropy intervals. 


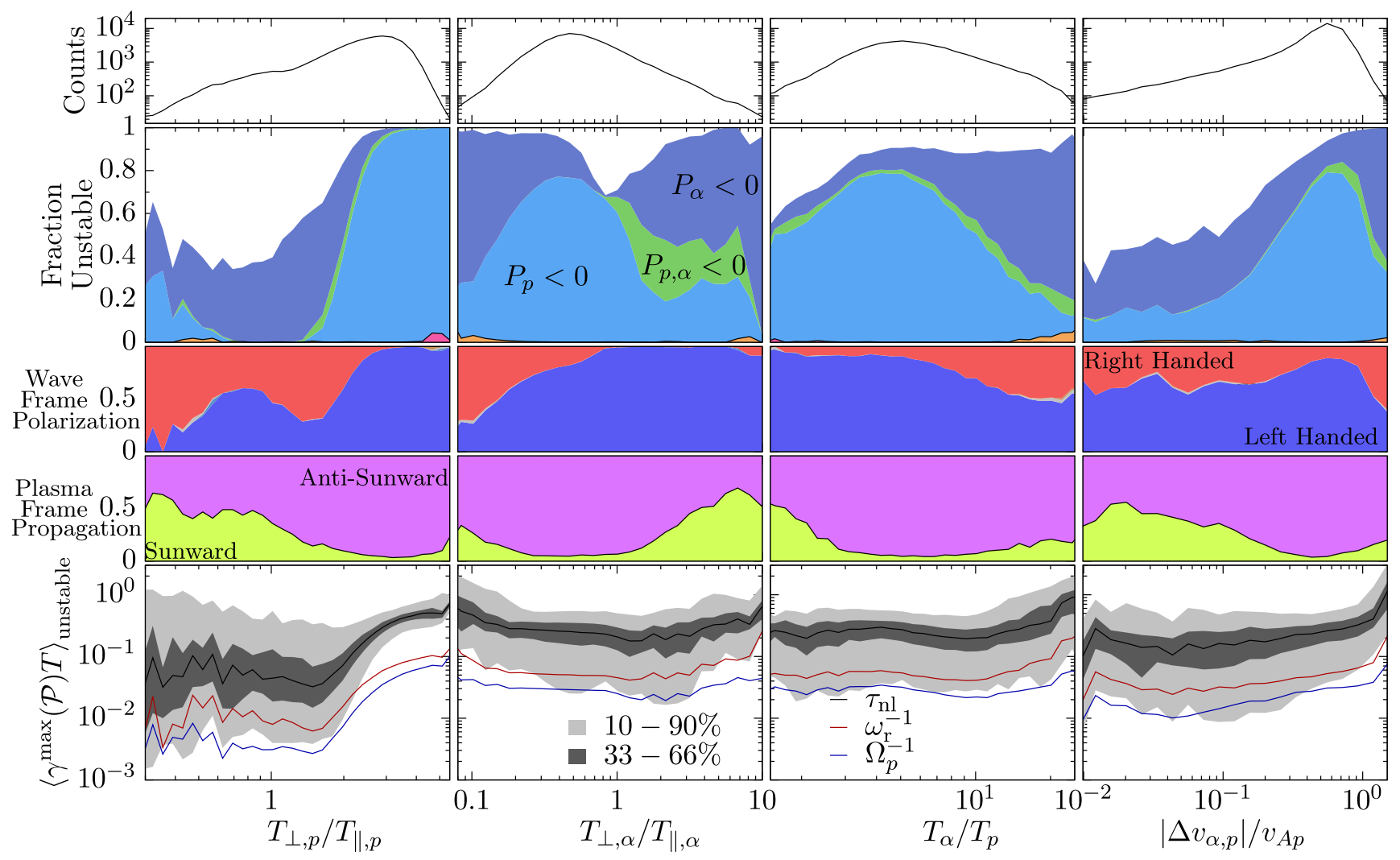

Figure 4. Statistical distributions characterizing the linear stability of the 45,147 intervals as a function of temperature anisotropies $T_{\perp, p} / T_{\|, p}$ and $T_{\perp, \alpha} / T_{\|, \alpha}$, scalar temperature disequilibrium $T_{\alpha} / T_{p}$, and normalized drift speed $\Delta v_{\alpha, p} / v_{A, p}$. The layout is described in the text.

The median growth rates are relatively constant with respect to $T_{\perp, \alpha} / T_{\|, \alpha}$. Departure from $T_{\perp, \alpha}=T_{\|, \alpha}$ leads to an increase in the fraction of unstable modes. Intervals with $T_{\perp, \alpha}>T_{\|, \alpha}$ are dominantly left-handed, with an admixture of sunward and anti-sunward waves; a significant fraction of these intervals have both the protons and $\alpha s$ emitting power. Intervals with $T_{\perp, \alpha}<T_{\|, \alpha}$ can be right- or left-handed.

Intervals with $T_{\alpha} \gg T_{p}$ have the $\alpha$ component as the primary power emitter. As the ion-temperature ratio decreases from $T_{\alpha} \gg T_{p}$ to $T_{\alpha} \gtrsim 4 T_{p}$, the protons drive a larger fraction of the instabilities, though $90 \%$ of the intervals remain unstable. Below $T_{\alpha} / T_{p} \approx 4$ the fraction of unstable intervals decreases. The median growth rate is fairly constant regardless of $T_{\alpha} / T_{p}$, with a slight uptick for $T_{\alpha} / T_{p}>10$, though intervals with such extremely large disequilibrium are relatively rare.

Intervals with relatively weak drift speeds, $\Delta v_{\alpha, p} / v_{A, p} \lesssim 0.1$, are less unstable than intervals with faster drift speeds, both in terms of fraction of intervals unstable and the median growth rate. For the slower drifting cases, both $\alpha$ s and protons contribute to the instabilities, and approximately equal admixtures of right- and left-handed and sunward and antisunward waves can be found. As the normalized drift speed grows toward unity, the unstable waves become preferentially left-handed with anti-sunward propagation, with the protons as the main driver of unstable growth. This changes for $\Delta v_{\alpha, p} / v_{A, p} \gtrsim 1.0$, where more of the unstable waves are righthanded, the $\alpha$ s contribute significantly to unstable growth, and the median growth rate increases significantly.

We next calculate the median growth rate $\gamma^{\max } / \omega_{\mathrm{r}}(\mathcal{P})$ as a function of two dimensionless parameters, $\beta_{\|, p}$ and $T_{\perp, p} / T_{\|, p}$. In Figure 5, the data are further subdivided into groups based on $v_{\mathrm{sw}}, R$, or $N_{\alpha, p}$. Unlike in Figures 3 and 4 , the calculation of the median value in Figures 5 and 6 includes both stable and unstable intervals, allowing the determination of whether a typical interval at a particular point in parameter space is stable or unstable. As found in Matteini et al. (2007), faster wind has larger proton temperature anisotropies and lower $\beta_{\|, p}$, while slow wind is more widely distributed throughout the $\left(\beta_{\|, p}, T_{\perp, p} / T_{\|, p}\right)$ plane. We see a spreading of the observed parameter distribution from low $\beta_{\|, p}$ and high $T_{\perp, p} / T_{\|, p}$ toward higher $\beta_{\|, p}$ and lower $T_{\perp, p} / T_{\|, p}$ with increasing distance, though as seen in Figure 3 the fraction of unstable intervals remains fairly constant with $R$. Observations that do not surpass the marginal stability thresholds for proton temperature anisotropy, as previously reported in Marsch et al. (2004), Matteini et al. (2007), and Stansby et al. (2019) using Helios data, and as seen at $1 \mathrm{au}$ (Kasper et al. 2002; Hellinger et al. 2006; Bale et al. 2009 ), are driven by $\alpha$-associated sources of free energy.

The variation with Coulomb number is striking. For collisionally young intervals, $N_{\alpha, p}<0.1$, typical values for $T_{\perp, p} / T_{\|, p}$ are larger, and the typical interval is unstable regardless of $\beta_{\|, p}$ and $T_{\perp, p} / T_{\|, p}$. For collisionally old intervals, $N_{\alpha, p}>0.1$, unstable intervals are much less common, with the median interval being stable.

To better constrain the role of collisional processing in the evolution of the solar wind, we calculate the median growth rate $\gamma^{\max } / \omega_{\mathrm{r}}(\mathcal{P})$ as a function of $N_{\alpha, p}$ and $v_{\mathrm{sw}}, R, \Delta v_{\alpha, p} / v_{A p}$, $T_{\perp, p} / T_{\|, p}, w_{\|, p} / c$ (a proxy for the proton temperature $T_{\|, p}$ ), and $T_{\alpha} / T_{p}$, as shown in Figure 6. We see the same pattern in all the joint distributions: for collisionally young solar wind with $N_{\alpha, p} \lesssim 0.1$ the median interval is linearly unstable, with relatively robust growth rates of $\gamma^{\max } / \omega_{\mathrm{r}}(\mathcal{P}) \gtrsim 3 \times 10^{-2}$. For collisionally older wind, most intervals are linearly stable. 


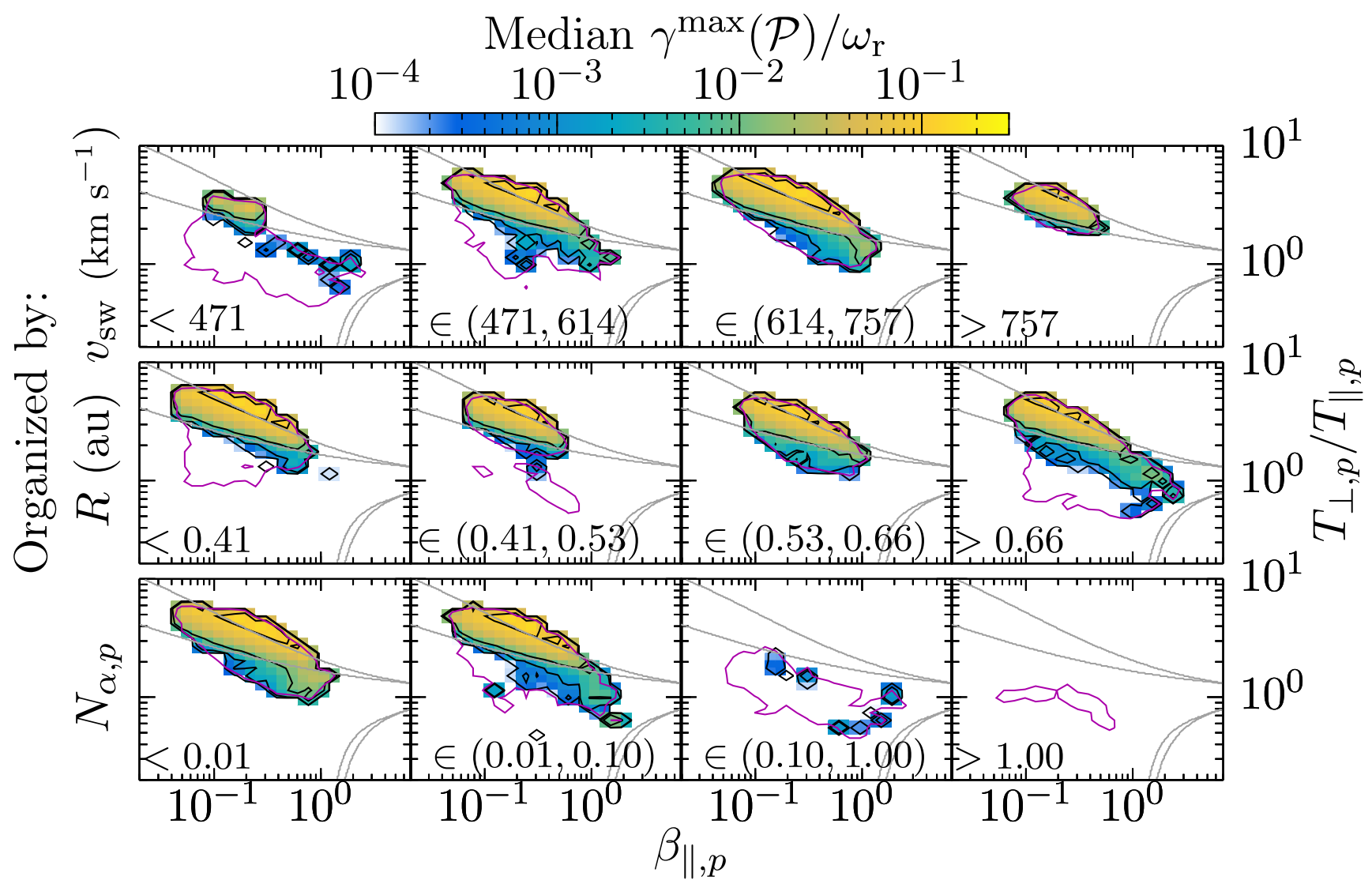

Figure 5. Median growth rate $\gamma^{\max } / \omega_{\mathrm{r}}$ as a function of $\beta_{\|, p}$ and $T_{\perp, p} / T_{\|, p}$ for subsets of the overall data subdivided into ranges of $v_{\mathrm{sw}}$ (top row), $R$ (center), or $N_{\alpha, p}$ (bottom). The pink lines indicate regions for which there are at least 10 intervals falling into the histogram bin. The gray lines represent the proton temperature anisotropy marginal stability thresholds for $\gamma / \Omega_{p}=10^{-2}$ taken from Verscharen et al. (2016).

From these panels, we also clearly see that the intervals with the hottest $\alpha$ s and the largest drift speeds are associated with the lowest values of $N_{\alpha, p}$.

\section{Conclusions}

The fraction of unstable intervals found in this data set of 45,147 Helios observations mostly drawn from the fast solar wind, $87.9 \%$, is larger than that reported from a much smaller set of 309 observations from the Wind spacecraft at $1 \mathrm{au}$, $53.7 \%$. The larger number of observations in the processed Helios data set enables a detailed statistical study of the parametric dependence of linear instabilities in the solar wind. We find that the occurrence rate of instabilities does not drastically change from 0.3 to $0.7 \mathrm{au}$, but that the nature of the instabilities changes with increasing distance, with the $\alpha$ component playing a more significant role in driving unstable modes at larger distances. The median growth rates $\gamma^{\max }(\mathcal{P})$ are relatively constant with $R$, with $\gamma^{\max }(\mathcal{P}) \tau_{\mathrm{nl}} \sim 0.3$, indicating that the growth rates of the instabilities are a non-negligible fraction of the turbulent cascade rate at $k^{\max } \rho_{p}$, and thus instabilities may grow quickly enough to impact the turbulent cascade.

It is clear that collisional processing plays an important role in modifying the particle velocity distributions and reducing the occurrence of linear instabilities, seen in Figures 3, 5, and 6. The continued presence of $\alpha$-driven instabilities for values of $N_{\alpha, p} \gtrsim 0.1$ while there are relatively few proton-driven instabilities may be indicative of the faster proton-proton collision rate removing sources of proton free energy more rapidly than $\alpha$-proton collisions.

It is unclear from these results how this dependence on collisions is compatible with the isotropization that should accompany linear wave-particle instabilities, seen for instance in nonlinear simulations (Hellinger et al. 2003; Hellinger \& Trávníček 2008), though evidence for combined action of collisions and instabilities has arisen from quasilinear models (e.g., Yoon et al. 2019). One conjecture could be that as the instabilities return the velocity distributions to marginal stability, finite-amplitude fluctuations could reignite unstable behavior, the so-called fluctuating anisotropy effect (Verscharen et al. 2016). This oscillation between unstable and stable states would only be arrested by the much slower process of collisional isotropization, which brings the equilibrium distribution closer to a Maxwellian state, requiring larger amplitude fluctuations to push the plasma unstable. Ongoing turbulent heating leading to increased perpendicular temperatures (e.g., Hellinger et al. 2013; Matteini et al. 2013) may also play a role in keeping distributions pinned near marginal stability in the expanding solar wind.

We emphasize that the processing of the data set of Stansby et al. (2019) intentionally did not include a proton beam component; this lack of a proton beam may underestimate the excess parallel pressure in the system, and may significantly impact a given interval's stability. Klein et al. (2018) found at 1 au that $68.8 \%$ of intervals for which a proton core, beam, and $\alpha$ component were resolved were unstable, while only $29.0 \%$ of intervals with only a proton core and $\alpha$ component were 


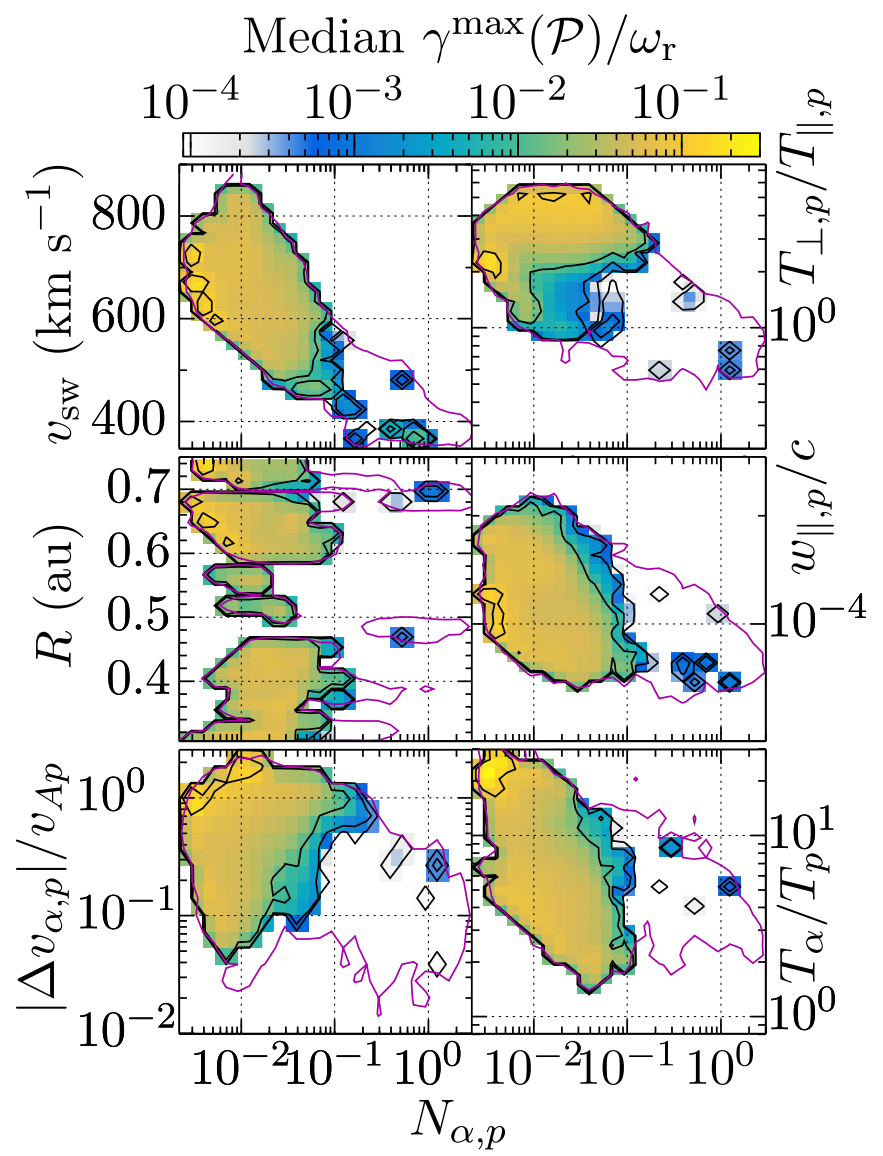

Figure 6. Median growth rate $\gamma^{\max } / \omega_{\mathrm{r}}(\mathcal{P})$ as a function of Coulomb number $N_{\alpha, p}$ and solar wind speed $v_{\mathrm{sw}}$ (top left), radial distance $R$ (middle left), ion drift speed (bottom left), proton temperature anisotropy $T_{\perp, p} / T_{\|, p}$ (top right), normalized proton thermal speed $w_{\|, p} / c$ (middle right), or ion temperature disequilibrium $T_{\alpha} / T_{p}$ (bottom right).

unstable. Extending this instability analysis to large sets of measurements that characterize the proton core, proton beam, and $\alpha$ components at $1 \mathrm{au}$, in the inner heliosphere (e.g., the recently reprocessed ion data set from Durovcová et al. 2019), and at much closer radial distances (e.g., with the Parker Solar Probe, Fox et al. 2015), will be necessary to improve our understanding of wave-particle interactions in the inner heliosphere.

Extending our analysis to include departures of the velocity distribution from a bi-Maxwellian model (e.g., Verscharen et al. 2018) will be essential to determining whether the solar wind actually supports these instabilities, or whether many of them would be suppressed if realistic phase-space densities are used in the calculation of the plasma response (e.g., Isenberg 2012).

The authors thank the referee for comments on the submitted manuscript. K. Klein is supported by NASA ECIP Grant 80NSSC19K0912. D. Stansby is supported by STFC grant ST/ S000240/1. T. Horbury is supported by STFC grant ST/ S000364/1. An allocation of computer time from the UA Research Computing High Performance Computing at the University of Arizona is gratefully acknowledged.

\section{ORCID iDs}

Kristopher G. Klein (1) https://orcid.org/0000-0001-6038-1923 Mihailo Martinović (ib https://orcid.org/0000-0002-7365-0472 David Stansby (1) https://orcid.org/0000-0002-1365-1908 Timothy S. Horbury (1) https://orcid.org/0000-0002$7572-4690$

\section{References}

Bale, S. D., Kasper, J. C., Howes, G. G., et al. 2009, PhRvL, 103, 211101 Chen, C. H. K., Matteini, L., Schekochihin, A. A., et al. 2016, ApJL, 825, L26 Duurovcová, T., Šafránková, J., \& Němeček, Z. 2019, SoPh, 294, 97

Feldman, W. C., Asbridge, J. R., \& Bame, S. J. 1974, JGR, 79, 2319

Fox, N. J., Velli, M. C., Bale, S. D., et al. 2015, SSRv, 204, 7

Gary, S. P. 1993, Theory of Space Plasma Microinstabilities (Cambridge: Cambridge Univ. Press)

Gary, S. P., Li, H., O'Rourke, S., \& Winske, D. 1998, JGR, 103, 14567

Goldreich, P., \& Sridhar, S. 1995, ApJ, 438, 763

Hellinger, P., Trávníček, P., Kasper, J. C., \& Lazarus, A. J. 2006, GeoRL, 33, 9101

Hellinger, P., Trávníček, P., Mangeney, A., \& Grappin, R. 2003, GeoRL, 30,1211

Hellinger, P., \& Trávníček, P. M. 2008, JGR, 113, 10109

Hellinger, P., \& Trávníček, P. M. 2014, ApJL, 784, L15

Hellinger, P., Trávníček, P. M., Štverák, Š., Matteini, L., \& Velli, M. 2013, JGR, 118, 1351

Hernandez, R., Livi, S., \& Marsch, E. 1987, JGR, 92, 7723

Isenberg, P. A. 2012, PhPl, 19, 032116

Kasper, J. C., \& Klein, K. G. 2019, ApJL, 877, L35

Kasper, J. C., Klein, K. G., Weber, T., et al. 2017, ApJ, 849, 126

Kasper, J. C., Lazarus, A. J., \& Gary, S. P. 2002, GeoRL, 29, 1839

Klein, K. G. 2013, PhD thesis, Univ. Iowa Zenodo, doi:10.5281/zenodo.50471

Klein, K. G., Alterman, B. L., Stevens, M. L., Vech, D., \& Kasper, J. C. 2018, PhRvL, 120, 205102

Klein, K. G., \& Howes, G. G. 2015, PhPl, 22, 032903

Klein, K. G., Howes, G. G., \& Tenbarge, J. M. 2017a, JPIPh, 83, 535830401 Klein, K. G., Kasper, J. C., Korreck, K. E., \& Stevens, M. L. 2017b, JGRA, 122,9815

Krauss-Varban, D., Omidi, N., \& Quest, K. B. 1994, JGR, 99, 5987

Kunz, M. W., Schekochihin, A. A., Chen, C. H. K., Abel, I. G., \& Cowley, S. C. 2015, JPIPh, 81, 325810501

Mallet, A., Schekochihin, A. A., \& Chandran, B. D. G. 2015, MNRAS, 449, L77

Marsch, E. 2012, SSRv, 172, 23

Marsch, E., Ao, X. Z., \& Tu, C. Y. 2004, JGRA, 109, A04102

Maruca, B. A., Chasapis, A., Gary, S. P., et al. 2018, ApJ, 866, 25

Maruca, B. A., Kasper, J. C., \& Gary, S. P. 2012, ApJ, 748, 137

Matteini, L., Hellinger, P., Goldstein, B. E., et al. 2013, JGRA, 118, 2771

Matteini, L., Landi, S., Hellinger, P., et al. 2007, GeoRL, 34, L20105

NASA 2018, Coordinated Data Analysis Web (CDAWeb), https://cdaweb. gsfc.nasa.gov/index.html/

Neugebauer, M. 1976, JGR, 81, 78

Nyquist, H. 1932, BSTJ, 11, 126

Quataert, E. 1998, ApJ, 500, 978

Schwartz, S. J., Burgess, D., \& Moses, J. J. 1996, AnGeo, 14, 1134

Stansby, D., Perrone, D., Matteini, L., Horbury, T. S., \& Salem, C. S. 2019, A\&A, 623, L2

Stansby, D., Salem, C., Matteini, L., \& Horbury, T. 2018, SoPh, 293, 155

Stix, T. H. 1992, Waves in Plasmas (New York: AIP)

Verscharen, D., Bourouaine, S., Chandran, B. D. G., \& Maruca, B. A. 2013, ApJ, 773, 8

Verscharen, D., Chandran, B. D. G., Klein, K. G., \& Quataert, E. 2016, ApJ, 831,128

Verscharen, D., Klein, K. G., Chandran, B. D. G., et al. 2018, JPIPh, 84, 905840403

Verscharen, D., Klein, K. G., \& Maruca, B. A. 2019, LRSP, 16, 5

Yoon, P. H. 2017, RvMPP, 1, 4

Yoon, P. H., Seough, J., Salem, C. S., \& Klein, K. G. 2019, PhRvL, 123, 145101 